anderweitige Störung der Augen, liest jetzt täglich Abends Stunden lang ohne alle Beschwerden, während er dies vor der galvanischen Behandlung nicht während weit kürzerer Zelt thun konnte.

Ich bin völlig überzeugt, dass die galvanische Behandlung nicht nur bei beginnendem Staar, sondern auch bei den meisten chronischen Krankheiten in dem Innern des Auges vom grössten Nutzen ist, und kann sie den Ophthalmologen von Fach auf's Angelegentlichste empfehlen. Hinsichtlich ihrer Wirkungsart will ich auf das an genannter Stelle Auseinandergesetzte verweisen.

\title{
XXII.
}

\section{Beitrag zur Functionslehre des weichen Gaumens und des Pharynx.}

Von Dr. R. Falkson,

Assistenzarzt an der chirurgischen Universitäts-Klinik zu Königsberg i. Pr.

(Hierzu Taf. VI.)

Vor $3 \frac{1}{2}$ Jahren wurden an der hiesigen chirurgischen Klinik von Herrn Collegen Gentzen') an einer Kranken, der durch Ausräumung der Augenhöble der Nasenrachenraum freigelegt war, Beobachtungen über die Bewegungen des Velum palatinum angestellt, wie sie in der Exactheit noch nicht bekannt geworden waren. Gerade zur Zeit, als ich mich mit der Lectüre des jüngst von Voltolini ${ }^{2}$ ) veröffentlichten Werkes über Rhinoscopie und Pharyngoscopie beschäftigte, führte der Klinik ein günstiger Zufall eine Patientin zu, an der in ganz analoger Weise wie in dem Gentzen'schen Falle Beobachtungen gemacht werden konnten. Nachdem mir durch Herrn Prof. Schoenborn in dankenswerthester Weise die Kranke zur Verfügung gestellt war, zögerte ich auch nicht einen Augenblick, die immerhin seltene Gelegenheit wahrzunehmen, zumal da Voltolini doch nicht zu ganz gleichen Resultaten gekommen war.

1) Beobachtungen am weichen Gaumen nach Entfernung einer Geschwulst in der Augenhöhle. Königsberg 1876.

2) Voltolini, Die Rhlnoscopie u. Pharyngoscopie. 2. Aufl. Breslau 1879. 


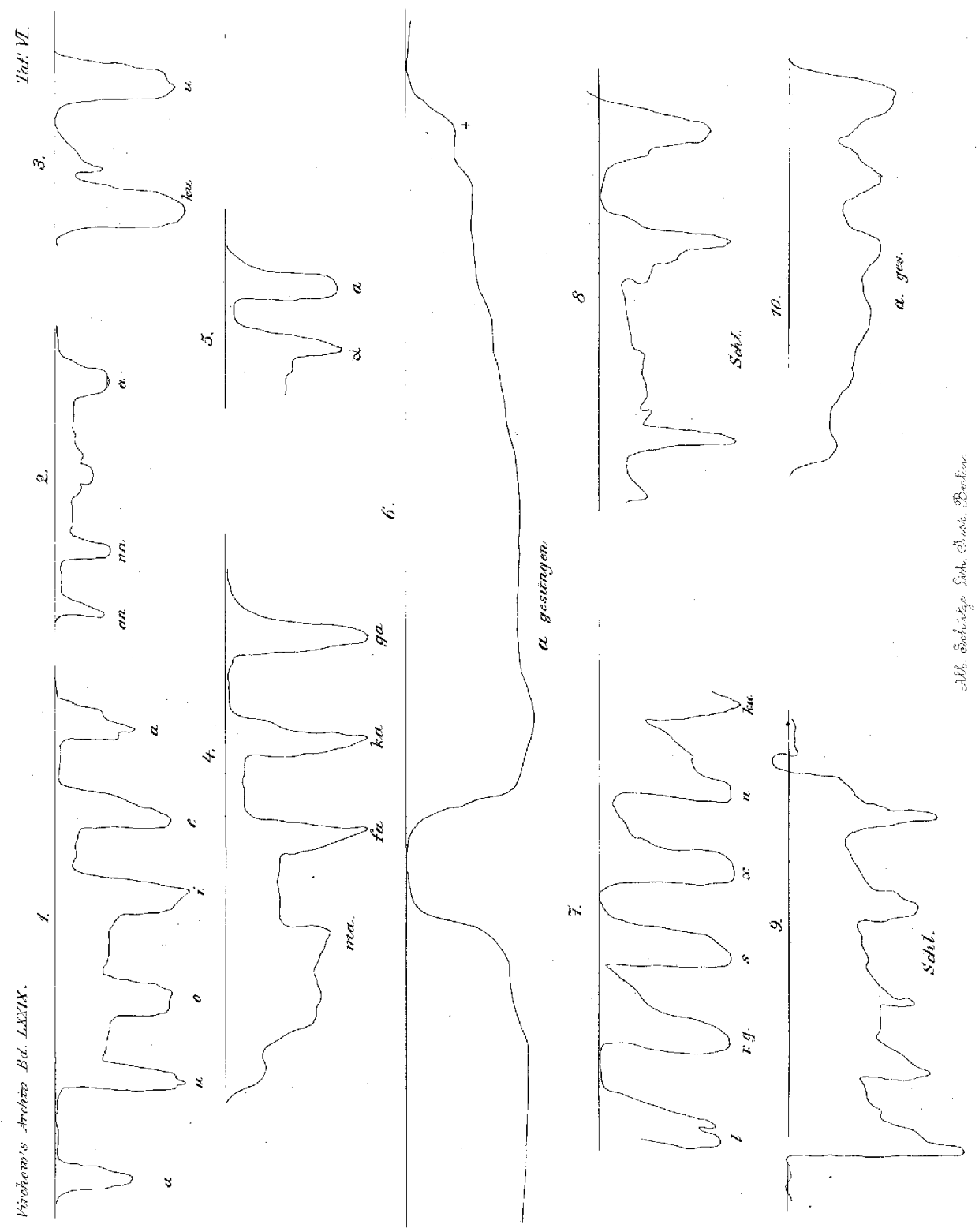


Auch in meinem Falle handelte es sich um ein Carcinom der Orbita, zu dessen Entfernung eine ausgiebige Operation nothwendig war, bei der weit über die physiologischen Grenzen der Augenhöhle hinausgegangen werden musste.

Wilhelmine D., 56 Jahre alt, wandte sich Ende October a. c. an die Klinlk, um von einem Uebel befreit zu werden, das thr das Leben anfing unerträglich zu machen. Von einem Naerus des rechten unteren Lides ausgehend hatte im Laufe von 3 Jahren ein derartiger deletärer Prozess um sich gegriffen, dass nunmehr von einer dauernden Heilung kaum die Rede sein konnte, doch berechtigten die grossen Schmerzen, die die Patientin Tag und Nacht peinigten, und der ekelhafte Gestank, durch den die Frau ihrer Umgebung sehr lästig wurde, binreichend, dennoch einen Versuch der Exstirpation zu machen, wenn nicht anders, um der Euthanasie Rechnung zu tragen. Das Carcinom hatte allmăhlich belde Lider zerstört, war dann auf den Augenhöhleninhalt und auf die knöchernen Wandungen übergegangen, nur der Bulbus hatte einigen Widerstand geleistet, wenn auch frühzeitige Erblindung eingetreten war, thn sah man in der Tlefe der Höhle als theils gelbliche theils röthliche Kugel in kaum noch erkennbarem Habitus bei Bewegungen des gesunden Auges kleine Excursionen machen.

Die Operation wurde am 28. October von Herrn Prof. Schënborn vorgenommen, es bam dabel am hinteren Theil des Daches der Orbita zu einer spaltförmigen Eröffnung der Schädelhöhle, aus der eine nicht unbedeutende Menge Gerebrospinalflüssigkeit aussickerte, ausserdem traten mehrere Schleimpolypen aus den Sinus frontales und dem Antrum Highmori hervor, die extrabirt wurden. Vom knöchernen Orbitalrand mussten überall kleine Partien abgetragen werden, ebenso von den Wünden der Augenhöhle, wo meistens allerdings der scharfe Löffel genügte. Zur Eröffnung des Antrum Highmori kam es nur in geringer Ausdehnung, dagegen geschah der rechten Nasenhöhle erhebliche Einbusse, es wurden hier das rechte 0 s nasi, die Lamina papyracea ossis ethmoidei mit zugehörigen Zellen und Muscheln, das Thränenbein und der Proc. nasal. maxill. sup. fortgenommen, kurz es wurde die mediale Orbitalwand bis auf das Septum nar. entfernt; nur der untere Nasengang blieb verschont. So war denn der grösste Theil der rechten Choane freigelegt, durch die man recht bequem die hintere Pharynxwand and das emporsteigende Velum palatinum inspiciren konnte. Zur Besichtigung des grössten Thells der Seitenwand des Pharynx, sowie der Tuba Eustachii mussten Hüllsmittel angewandt werden. Pharynx und Palatum molle schienen vollkommen normal, nur die Verbältnisse des Ostium pharyng. tubae schienen ungewöhnlich, worauf lch später zurückkommen werde.

Der Wundverlauf war trotz der ausgedehnten Infectionsmöglichkeit durch Rachenund Nasenhöhle ein vollkommen aseptischer (der Verband bestand in einem einfachen Carbolgazetampon), ebenso blieben meningitische Erscheinungen aus. Nachdem die Granulationsbildung einigermaassen im Gange, begann ich am 17. November meine Untersuchungen. 
Beim Sprechen, Schlucken, Husten, bei tiefen Inspirationen etc. sah ich jedes Mal Bewegungen des weichen Gaumens und Schlundkopfes mil grosser Deutlichkeit eintreten und sich die Raumverhältnisse des Nasenrachenraumes verändern.

Ich musste grösstentheils meine Untersucbungen bei künstlicher Beleuchtung vornehmen und benutzte zur Erhellung des Gesichtsfeldes einen gewöhnlichen Stirnreflector, zu specielleren Zwecken führte ich einen kleinen Kehlkopfspiegel in den Nasenrachenraum ein, als sehr zweckmässig erwies es sich auch, der Kranken den Kopf stark hintenüber zu beugen und Licht von oben her (Hängelampe) auf die zu untersuchenden Theile fallen zu lassen, concentrirte ich nun das Licht durch eine in einem kurzen Tubus befindliche Sammellinse, so konnte ich, hinter der Patientin stehend, weit in den Rachen hineinsehen, ja es war sogar möglich, im günstigen Augenblick und bei günstigem Lichteinfall die Epiglottis zu sehen.

Ausserdem benutzte ich den von Gentzen angegebenen Hebelapparat, den ich mir nach dessen Angaben nachconstruiren liess, leider fiel er aber schwerer aus, wodurch die Feinheit der Aufzeichnungen etwas leidet, wenn auch die wesentlichen Erfordernisse in derselben Weise erfïllt werden, schwerer deshalb, weil sich mein Lieferant stalt des Aluminiumdrabtes, den er in der erwünschten Eile nicht schaffen konnte, eines nicht allzuzarten Messingdrahtes bediente, dieses Uebel wird aber jedem gering erscheinen, der die relativ immense Muskelkraft des Gaumensegels kennen gelernt hat, was auch Gentzen 1. c. S. 17 betont. Es ist ein zweiarmiger Hebel, der an seinem kurzen Arm ein Gewicht trägt (Silber- oder Messingplättchen), das seiner Schwere nach dem Velum aufliegt, der lange Arm trägt den Zeiger mit der Schreibfeder, der Drehpunkt kommt vor der Mitte der Choane zu liegen und besteht aus einem feinen Zapfen, der wiederum durch einen horizontalen Metallstab sichere Stellung erhält, letzterer wird durch Vermittelung eines mit ihm durch eine Klemme verbundenen verticalen Stabes und Stirnbinde an der Stirn in fester Stellung gehalten (nähere Angaben cf. Gentzen 1. c. S. 9, 10, 16-18). Der Draht ist so gebogen, dass er nirgends bei den Excursionen des Zeigers anstossen kann ${ }^{1}$ ). Der Kopf der Patientin wurde bei den Versuchen durch einen Stützapparat,

1) Um Missverständnisse zu vermeiden, will ich hier bemerken, dass ein Fallen des Zeigers einem Heben des Velum entspricht. 
wie ihn die Photographen haben, fixirt. Zum Aufzeichnen der Curven benutzte ich anfangs eine rotirende Trommel, hierbei machte sich aber die Kreisbewegung der Zeigerspitze in so störeuder Weise geltend, dass ich nach mehrtägigen vergeblichen Versuchen zum Marey'schen Sphygmographen überging. Da man bei der Trommel nur immer eine senkrechte Linie des Cylindermantels in Betracht ziehen kann, so kommt es immer bei der Grösse der Excursionen streckenweise zu einer Abhebung des Zeigers vom Apparat. Vermeiden lässt sich dies nur durch Verkürzung der Excursionen durch Verkürzung des Hebels, was der verschiedenen Prominenzen des Gesichtes wegen nicht möglich, oder man drückt den Zeiger recht fest gegen den rotirenden Cylinder, dann kommt man aber $\mathrm{zu}$ sehr plumpen und deshalb auch ungenauen Curven. Um diesem Uebel abzuhelfen dürfte vielleicht nur eine kleine Aenderung nöthig gewesen sein, aber die Zeit oder vielmehr die Patientin drängte und so konnte ich mich mit Probiren nicht lange aufhalten.

Von grossem Einfluss auf die absolute Höbe der Curven ist die Lage der Pelotte (des Gewichtes) auf dem weichen Gaumen, je tiefer dieselbe zu liegen kommt, desto grösser sind die Bewegungen des Zeigers, das relative Verhältniss wird allerdings dabei wenig leiden; wichtiger ist es, die Pelotte möglichst nahe der Mittellinie anzubringen, denn bei mehr seitlicher Lage kommt zu der einfach auf- und absteigenden Bewegung noch die Seitenversehiebung in Betracht und dieser Umstand macht die bezüglichen Curven geradezu werthlos. Die Seitenverschiebung macht sich dabei so deutlich bemerkbar, dass es für den Beobachter unschwer ist, die brauchbaren von den unbrauchbaren Curven zu sondern. Es ist deshalb auch von keinerlei Bedeutung, wenn meine Curven niedriger sind als die Gentzen'schen, um so weniger da meine Patientin ein ziemlich kurzes Velum hatte, keinesfalls aber ein pathologisch verkürztes oder zu kurzes (dem entsprechend war auch der ganze Nasenrachenraum etwas eng).

Bemerken will ich noch, dass die Sprache der Patientin eine etwas näselnde war, scharf und deutlich zu sprechen war ihr auch nur bis zu einem bescheidenen Maasse beizubringen, da sie zu alt und ungebildet war, um neue Lehren anzunehmen, dazu noch einen leichten Anflug von Grössenwahn besass, so brachte ich sie auch nicht so weit, einigermaassen brauchbar zu "lautiren“, wie es jetzt 
in jeder Schule gelehrt wird, und nur mit Mühe gewöhnte ich es ibr ab, jeden Buchstaben, den sie phonirte, durch ein Kopfnicken zu bekrö̌ftigen.

Bei der Sprache betheiligt sich der weiche Gaumen in $\operatorname{ganz}$ typischer Weise, es ist schon deshalb die Annahme unerlaubt, dass es hier sich nur um eine untergeordnete mechanische Mitwirkung handelt (Bidder). Bei jedem Worte, bei jedem Buchstaben, den Patientin sprach, erhob sich das Gaumensegel und wurde, während es sonst sich dem directen Einblick entzog, in seiner ganzen rechten Hälfte, bei Seitwärtsdrehung des Kopfes auch in einem Theil der linken Hälfte sichtbar, gleichzeitig rückten die Seitentheile des Pharynx einander näher und trat die hintere Pharynxwand bervor, der Passavant'sche Querwulst ${ }^{1}$ ) war dabei nur schwach entwickelt. Dieser letzte Umstand beeinträchtigt durchaus nicht die Wichtigkeit des Querwulstes, er zeigt nur, dass ebenso wie andere Muskeln, auch der Constrictor pharyng. sup. bei einem Menschen schwächer entwickelt sein kann als beim anderen. Auch eine Längsfaltung konnte ich constatiren und zwar am besten, wenn jch, wie oben erwähnt, die Augenhöhle bei hintenübergebeugtem Kopf von oben ber erleuchtete und hinter der Patientin stehend beobachtete. Ich sah hier 3 Längswülste an der hinteren Schlundkopfwand, einen medialen und 2 laterale, dem entsprechend 4 Furchen, von denen die äussersten den Uebergang in die Seitentheile des Pharynx vermittelten, demnach entspricht der mittlere Wulst dem von Michel ${ }^{2}$ ) angegebenen ,etwa $1 \mathrm{Cm}$. breiten glatten Zwischenraum", der in der That bei der Inspection von vorn scheinbar vorhanden ist. Nach oben, dem Ansatze des Pharynx, verlieren sich natürlich Furchen und Wülste. - Am wenigsten hebt sich das Gaumensegel bei a, dann bei $e$ und $o$ und am höchsten bei $i$ und $u$, bei $u$ gewöhnlich etwas höher als bei $i$, dies stimmt nun mit den Befunden, wie sie Gentzen (1. c. S. 12) und Voltolini (1. c. S. 229) erhalten baben, überein. Bei den Consonanten war der Unterschied ein nicht so typischer, bei den Resonanten $m$ und $n$, die, ohne den zugehörigen Vocal zu nennen, meine Patientin hin und wieder fertig brachte, stand das Gaumensegel vollständig still. Bei den Verschluss-

1) Dieses Archiv 1869, 1. Heft erster Aufsatz und "Ueber die Verschliessung des Schlundes beim Sprechen “, Frankfurt a. M. 1863.

2) Berlin. klin. Wochenschr. 1875. No. 41. 
lauten stieg das Velum so hoch oder etwas höher wie bei $\mathfrak{u}$, bei den Reibungsgeräuschen erreichte es nicht ganz dieselbe Höhe, die Differenz war aber nur gering, wenn ich sie auch constant nacbweisen konnte. Bei $1, s$ und am meisten bei $r$ sah ich das Gaumensegel in leicht zitternde Bewegungen gerathen, zu deren Aufzeichnung allerdings mein Apparat nicht feinfühlend genug war, wie es der Gentzen'sche im hohen Grade (cf. l. c. Curve M); meine Patientin konnte nur das gutturale $\mathbf{r}$ brauchbar aussprechen.

Während bei a das Velum kaum die Horizontale erreicht, erhebt es sich bei den übrigen Buchstaben (mit Ausnahme von $\mathrm{m}$ und $n$ ) über dieselbe.

In analoger Weise waren auch die Bewegungen der Pharynxwände und demgemäss auch die Verengerung des Nasenrachenraumes.

Da die Kranke nicht lautiren konnte, so liess ich sie, um die Consonanten unter gleiche Verhältnisse zu setzen z. B. nicht ge und ka, sondern ga und ka sagen. Sprach Patientin mehrere Buchstaben hintereinander, so fiel es mir auf, dass das Velum selbst bei möglichst getrennter Aussprache nicht nach jedem Laut in die Ruhestellung zurückkehrte, sondern nur bis zu einem gewissen nicht constanten Grade sank; ich glaube, diese Erscheinung als senile Steifigkeit und Trägheit auffassen zu müssen, wie es ja auch bei dieser Frau, die reichlich 10 Jahre älter aussah, als sie in Wirklichkeit war, leicht denkbar.

Dass ich bei meinen Curven nicht die Schwankung des weichen Gaumens beim Phoniren der Vocale aufzeichnen konnte, wie wir es bei Gentzen bei a z. B. (cf. Curve A, G, N, 0) als Regel finden, liegt wohl einmal an der grösseren Unvollkommenheit meines Apparates, dann aber auch und wohl zum grössten Theil an der schlechten Aussprache der Patientin, der letzteren muss ich es ebenso zuschreiben, dass die Differenz in den Curven der einzelnen Vocale nicht die Höhe erreicht, wie wir sie bei Gentzen finden.

Die Trägheit des weichen Gaumens veranschaulicht deutlich die Curve $\mathbf{1}^{1}$ ). Die Thätigkeit des Gaumens beginnt rechts bei a, hir-

1) Ich muss hier darauf aufmerksam machen, dass nicht die verschiedenen Curven mit einander verglichen werden können, sondern nur Theile derselben Curve, da es ja, wie früher betont, nicht möglich ist, die Pelotte immer auf einen identischen Punkt des Velum zu setzen. 
ter a kehrt er wieder fast vollständig zur Ruhestellung zurück, wie sie die Horizontallinie andeutet, dann aber erst nach Phoniren des $u$, in der Zwischenzeit macht er nur zur Hälfte den Rückweg; Patientin hat ungefähr in derselben Geschwindigkeit gesprochen, wie die Gentzen's (ich habe denselben Sphygmographen benutzt) und doch genügte der Zeitraum zwischen dem Sprechen der einzelnen Vocale dazu nicht. Diese Langsamkeit der Rückkehr zur Ruhestellung ist natürlich nicht constant, wie gross dieselbe aber bei einiger Ermüdung sein kann, sieht man z. B. am Ende von Curve 4 (von rechts nach links zu betrachten) bei ma. Die Curve 2 zeigt die Einflusslosigkeit der Resonanten auf die Bewegung des weichen Gaumens; a, an und na macht absolut keinen Unterschied. Bei Curve 3 sehen wir, dass bei den Verschlusslauten das Gaumensegel höher als bei u steigen kann, wenn auch die Differenz nur eine geringe ist. Curve 4 giebt ein Beispiel zum Vergleich von Verschlusslauten und Reibungsgeräuschen, bei fa steigt das Gaumensegel nicht ganz so hoch wie bei ka und ga, während es bei ma nur die Höhe von a erreicht, dieselben Verhältnisse werden auch durch Curve 7 erhärtet ( $r$ g bedeutet $r$ gutturale), ku steht am höchsten (in der Curve, die die Stellung des Zeigers markirt, am niedrigsten), $u$, die Reibungsgeräusche $l$ and $s$, sowie der Zitterlaut $r$ und der zusammengesetzte Consonant $\mathrm{x}$ gleich hoch.

$\mathrm{Ob}$ ein Buchstabe mehr oder weniger laut oder gar geflüstert ausgesprochen wird, hat so gut wie gar keinen Einfluss auf die Höhenstellung des weichen Gaumens, als Beispiel dafür diene Curve 5, wo $\alpha$ ein geflüstertes a bedeutet, während a die Lautsprache repräsentirt, ich stimme daher vollkommen mit Gentzen tuberein, der S. 20 sagt: „Wenn mitunter kleine Schwankungen vorkommen, so hängen dieselben meiner Meinung nach nicht von der Intensität, sondern von der verschiedenen Reinheit und Deutlichkeit der Aussprache ab." Damit stimmt denn auch überein, dass bei meiner Patientin die Curve für $\mathbf{u}$ gewöhnlich gleich hoch wie die für i (ef. Curve 1) ausfiel, da dieselbe nicht $u$, sondern einen $Z$ wischenlaut zwischen $\mathrm{u}$ und $\mathfrak{a}$ sagte, wie man ihn hier in Ostpreussen öfter antrifft.

In einem Punkte bin ich allerdings nicht mit Gentzen einer Meinung und d. i. in Bezug auf den vollständigen Abschluss des Nasenrachenraumes vom Cavum pharyngolaryngeum beim Sprechen. 
Ein vollständiger Verschluss fand bei meiner Patientin nicht statt, am wenigsten bei a, am meisten bei $\mathrm{i}, \mathbf{u}$ und den Consonanten (ecl. $m$ und $n$ ). Bei a berührt aucb nicht die Uvula die hintere Pharynxwand, ich konnte z. B. zwischen Velum und hinterer Pharynxwand einen $2 \mathrm{Mm}$. dicken Spiegel zwischenlegen, obne dass der Klang von a irgend welchen Schaden erlitt, ja der Spiegel wurde nicht einmal von dem Velum anhaftendem Schleim benetzt, und dabei klang a sogar am wenigsten näselnd von allen Buchstaben. Liess ich die Patientin, wenn der Spiegel zwischen lag, i phoniren, so kam ein nicht ganz deutliches ich (ich gutturale) zu Stande, aber auch bei $i$ und $u$ war noch eine Communication zwischen oberem und unterem Theil des Rachens da, wie ich wiederholt durch Spiegeluntersuchung und durch Beleuchtung von oben (cf. früher) constatiren konnte. Die Verbindung wurde hier vermittelt durch einen auf beiden Seiten vorbandenen lateralen Spalt, der den seitlichen Grenzen der Wurzel der Uvula entsprach und mit dem zu Seiten des Mittellängswulstes der hinteren Schlundwand gelegenen feinen Längsspalt correspondirte. Die Spitze der Uvula wird oben natürlich nie sichtbar.

Diese Frage haben nun Bruecke ${ }^{1}$ ) und $\mathrm{Czermack}^{2}$ ) durch ihre Versuche mit dem Wachslicht und Metallspiegel zu entscheiden gesucht. In wie weit diese Experimente maassgebend sind, darüber hat sich Voltolini in extenso ausgesprochen und will ich, um unnütze Wiederholungen zu vermeiden, auf ihn verweisen (cf. l. c. S. 220-222), anfïhren will ich hier nur noch, dass auch die feuchtwarme Luft, die sich im Nasenrachenraum an und für sich befindet, Berücksichtiguńg verdient, bei jeder Hebung des Velum muss ein Theil derselben, wenn die Nasenhöhlen nicht verlegt sind, entweichen und dadurch kann schon allein ein mässiger Beschlag des Spiegels bedingt sein. Bei meiner Patientin beschlug der Spiegel jedes Mal beim Phoniren von Buchstaben, nackdem ich ihn mit möglichster Genauigkeit soweit erwärmt hatte, dass nicht in der Augenhöhle allein schon ein Beschlagen eintreten konnte.

Passavant und Gentzen haben den Verschluss direct nachzuweisen gesucht, ersterer hat an Patienten mit gespaltenem Gaumen Untersuchungen gemacht, die doch nicht wegen der patholo-

1) Grundzüge der Phys. und Syst. der Sprachlaute, S. 28.

2) Wiener akadem. Sitznngsberichte. Bd. 28. 
gischen Verhältnisse für Entscheidung dieser wichtigen Frage maassgebend sein dürtten, und Gentzen hat bei seinem Fall nicht den Spiegel zu Hülfe genommen oder nehmen können und bei der directen Inspection einen Verschluss gesehen, das genügt aber eben nicht, wie ich mich bei meiner Patientin für die Buchstaben $i$ und $\mathrm{u}$ wenigstens überzeugen konnte; bei a war allerdings die Spiegeluntersuchung nur als Controlle nothwendig. - Ferner bat man als maassgebend das Verhalten der Sprache angesehen, bei unvollständigem Verschluss sollte durchaus Näseln eintreten müssen. Nun hat Voltolini zu der altbekannten Thatsache, dass bei Defecten congenitaler oder acquirirter Natur im harten und weichen Gaumen, ebenso bei Lähmungen, die Sprache näselnd wird, gezeigt, dass auch bei vollständigem Abschluss derselbe Effect eintritt (auf Verstopfung der Nase durch entzündliche Prozesse oder durch Tumoren will ich nicht besonders hinweisen); er hat bei einem Patienten, bei dem wahrscheinlich durch senile Vorgänge (cf. l. c. S. 210) „das Gaumensegel durch die Arcus pharyngopalatini beinahe straff an der hinteren Rachenwand festgehalten" wurde, künstlich den absoluten $\mathrm{Ab}$ schluss des Cavum pharyngonasale vom Cav. pharyngolaryngeum durch Tampons ete. bewerkstelligt und darnach exquisit nasalen Klang der Sprache beobachtet. Gleiches sah Dr. Kuhn ${ }^{1}$ ) an einem Knaben, dem das Gaumensegel durch einen pathologischen Prozess an der hinteren Pharynxwand so angewachsen war, dass der Nasenrachenraum nach unten vollständig abgeschlossen wurde.

Diese Thatsachen beweisen wohl zur Genüge, dass die bisherige Erklärung der näselnden Sprache durchaus nicht hinreiche und also auch für meinen Fall nicht mit vollem Recht der Einwand gemacht werden kann, dass eben der unvollständige Verschluss die Ursache der etwas näselnden Sprache der Patientin sei, ausserdem waren am Pharynx und Velum durchaus keine derartigen Veränderungen, die etwa der regelrechten Wirkung der Muskeln entgegentraten, es waren keine Narben vorbanden, ebenso wenig bestand eine Spur von Lähmung. Sehr willkommen ist es mir aber dennoch, dass ich in den Voltolini'schen Beobachtungen, die an Gesunden angestellt sind, eine Stütze habe. Voltolini hat durch seine rhinoscopischen Experimente meiner Meinung nach in so genügender Weise

) von Voltolini S. 211 citirt. 
das Fehlen eines vollständigen Abschlusses erwiesen, dass ich, im Falle der Befund bei meiner Kranken ein anderer gewesen wäre, nur an der Beweisfähigkeit des Falles gezweifelt hätte.

Noch eine Erfahrung möchte ich zu Gunsten des oben besprochenen Factums anführen, d. i. der vollkommene Erfolg, wie er durch die Suersen'schen Obturatoren beim sogenannten Wolfsrachen in Bezug auf die Sprache erhalten wird. Ein vollständiger Abschluss im Sinne von Gentzen und Passavant kann durch dieselben unmöglich bewirkt werden, in den seitlichen Partien muss immer eine Communication zwischen Cavum pharyngonasale und -laryngeum da sein, das ergiebt sich ja klar aus der kugligen Begrenzung des Theiles des Obturators, der in den Rachen zu ljegen kommt.

Den unvollkommenen Abschluss sehe ich also als einen durchaus physiologischen Vorgang an, wie erklärt sich aber bei meiner Patientin anders die näselnde Sprache? Erwähnen muss ich noch, dass dieselbe stärker hervortrat, wenn der Tampon aus der Augenhöhle herausgenommen war, als wenn er in derselben steckt, im ersteren Falle communicirte der Naseurachenraum in pathologisch ausgedehntem Maasse mit der äusseren Luft, im zweiten waren dieselben Verbältnisse, wie bei einseitiger Verstopfung der Nase. Die Erklärung Voltolini's (S. 236), dass der Nasenton durch Mitschwingungen der Nasenknochen entsteht, genügt hier nicht, denn hier fehlt eben ein Theil der Nasenknochen, was doch das Entstehen des Nasentons erschweren musste, wenn nicht etwa die Knochen der Augenhöhle vicariirend eintreten, andererseits ist aber auch die Resonanz der Luft (alte Erklärung) in der Nasenhöhle nicht gerade begünstigt, ich weiss mich daher nicht anders aus dem Dilemma zu zieben, als indem ich die weite Communication zwischen Cavum pharyngonasale und Orbita für den Nasalklang verantwortlich mache, ob nun die Luft in der Höhle oder die begrenzenden Knochen die Klangfarbe bestimmen, wage ich nicht zu entscbeiden, jedenfalls war die Art des Nasalklanges bei herausgenommenem Tampon der Art, dass man, wenn Patientin sprach, sich sagen musste, sie treibt zu viel Luft durch die Nase (oder vielmehr durch die Orbita).

Ich komme nun zu der Betheiligung des Velum palatinum beim Singen. Zu behaupten, dass mit der Höhe des Tons auch die 
Höhenstellung des weichen Gaumens zunimmt, ist wohl nur bei ganz oberflächlicher Untersuchung möglich, da viele Menschen die Gewohnheit haben, beim Singen einer Octave z. B. mit der Höhe des Tons den Kopf höher zu heben, den Hals zu recken und die Schultern in die Höhe zu ziehen etc., d. h. alle auxiliatorischen Athemmuskeln in Thätigkeit zu setzen, was wohl auch bei schwierigen Tönen seine Berechtigung hat; bei genauerer Beobachtung sieht man aber, dass anfangs die Höhe des Tons gar keinen Einfluss auf die Stellung des Gaumensegels ausübt, wie es ja auch Gentzen (cf. S. 21, 22) beobachtet hat, später stellt sich regelmässig ein Sinken des Velum ein, nur glaube ich, diesem Umstand nicht die Bedeutung beilegen zu dürfen, die ihr Voltolini (l. c. S. 231) beigelegt hat. Letzterer beobachtete das successive Sinken regelmässig, gleichzeitig wurde aber auch der Abschluss des. Cavum pharyngonasale durch stärkeres Hervortreten des Querwulstes ein vollständigerer. Bei meiner Patientin war das nicht der Fall, der an und für sich wenig ausgebildete Querwulst wurde nicht deutlicher und ebenso der freie Raum zwischen Velum and Pharynx nicht enger, bei ihr lag das Sinken einmal in der ziemlich leicht eintretenden Ermüdung, dann aber auch darin, dass Patientin den Vocal, den sie singen sollte, immer weniger und weniger deutlich heraushören liess, darum scheint mir auch mein Fall für diese Frage nicht beweisend genug.

Das eben Gesagte veranschaulichen die Curven 6 and 10. Die Curve 6 ist auf eine rotirende Trommel gezeichnet und läuft von links nach rechts, anfangs steht das Gaumensegel fest auf dem Standpunkt, der ihm für a zukommt, sinkt alsdann zur Rubestellang zurück, indem Patientin von Neuem inspirirt und frisch einsetzt, stejgt dann wieder zur selben Höhe, um allmählich wieder zu sinken und am Ende der gesungenen Octave $(+)$ in die Nähe der Ruhestellung zu gelangen, in die es denn auch wegen der Kürze der zurückzulegenden Strecke steil zurücksinkt. Bei 10 sehen wir den Vorgang in ähnlicher Weise, hier markirt sich nur noch jeder höhere Ton (Verlauf umgekehrt wie bei 6) durch eine neue mehr oder weniger scharf markirte Curvenwelle.

Das Sprechen wird mehr oder minder unmöglich, wenn den Bewegungen des Gaumensegels ein kräftiger Widerstand geleistet wird, der Grad der Behinderung ist abhängig von der Ausdehnung der Function d.h. das Hinderniss macht sich um so mehr geltend, 
je höher das Gaumensegel gerade $z u$ steigen hat. Hält man das Gaumensegel mit Gewalt von oben her zurück, wie ich es in meinem Falle von der Augenhöhle aus mit einem starken metallenen Katheter that, so wird das Phoniren der einzelnen Buchstaben je nach der erforderlichen Excursion erschwert, ausserdem wurde bei meiner Patientin die Sprache näselnder; am undeutlichsten und z. Th. ganz unverständlich wurden die Consonanten, während a noch sehr gut zu verstehen war, es stimmte dies somit nicht mit Gentzen's Beobachtung (1. c. S. 17), dessen Patientin durch diesen Eingriff das Sprechen gänzlich unmöglich gemacht wurde. Wie gross die Kraft des Velum ist, mit der es den Widerstand zu überwinden sucht, ist übrigens erstaunlich und kann sich wohl schwerlich Jemand davon eine rechte Vorstellung machen, der nicht ein ähnliches Experiment angestellt hat; bei dem etwas langen Hebelarm des Katheters muss man mit voller Faust einen Druck ausüben, wenn nicht das Gaumensegel doch noch kleine Hebungen zu Stande bringen soll.

Von rein passiver Natur ist die Betheiligung des Velum palatinum beim Athmen. Liess ich Patientin ruhig durch den Mund oder Nase athmen, so konnte ich gar keine oder kaum nennenswerthe Bewegungen desselben beobachten, ebenso fehlten dieselben beim liefen In- oder Exspiriren durch die Nase. Athmete Patientin tief durch den weit geöffneten Mund, so erhob sich das Gaumensegel nicht ganz bis zur Horizontalen, blieb aber in dieser Stellung nur, wenn die Athmung sebr exact vollzogen wurde, um beim Schluss der Inspiration schnell herabzusinken, geschah die Inspiration aber absatzweise, was bei weitem das Gewöhnliche war, so machten sich diese Absätze, wenn sie auch noch so geringfügig waren, durch leichtes Sinken und wieder Heben des Velum sofort bemerklich. Bei tiefer Exspiration war der Einfluss auf den weichen Gaumen durch ein plötzliches Emporschleudern desselben bemerklich, dem aber schon vor Ende der Exspiration ein Herabsinken folgte, um diese Erscheinung hervorzubringen war aber schon sehr kräftige Exspiration nothwendig, daher war dies Spiel auch am deutlichsten beim Husten zu beobachten. Diese Bewegungen konnte ich leider nicht aufzeichnen, weil sich dabei die Schwere des kleinen Apparates sehr geltend machte. Es erscheint mir daher fraglos, dass die Betheiligung des weichen Gaumens beim Athmen eine rein passive und nebensächliche ist. 
Sehr hübsch konnte ich bei meiner Patientin die Erscheinungen beim Schnarchen beobachten. Sowohl beim inspiratorischen als exspiratorischen Schnarchen fand eine Hebung des Gaumensegels statt, die Hauptsache ist aber die flottirende, zitternde Bewegung, in die Uvula und die hinteren Gaumenbögen gerathen, ausserdem bäumt sich die Zungenwurzel auf und verengt so den Aditus faucium. Die Erhebuug des Gaumensegels ist grösser beim inspiratorischen als beim exspiratorischen Schnarchen und stimmt dies auch damil uiberein, dass das exspiratorische Schnarchen geringere Kraft erfordert. Dagegen spielt wieder beim exspiratorischen Schnarchen die Zungenwurzel eine grössere Rolle, als beim inspiratorischen, wie man auch leicht an sich selbst beobachten kann, drückt man nehmlich mit dem Finger oder Spatel die Zungenwurzel mit Gewalt herab, so kann man zwar noch ziemlich gut bei der Inspiration schnarchen, nicht die Spur aber exspiratorisch.

Ich komme nun zu der eigentlich wichtigsten Function des Pharynx und Gaumensegels d.i. zum Schlucken. Bei diesem Acte wird der Nasenrachenraum am meisten verengert und am vollkommensten vom Cavum pharyngolaryngeum abgeschlossen. Der Querwulst macht sich deutlich bemerkbar ebenso aber auch die Längsfaltung, die Seitenwände rücken einander näher denn je und das Gaumensegel wird hoch emporgehoben, so dass es deutlich eine nach oben convexe Fläche bildet, es macht sich auch an ihm eine gewisse Faltung bemerklich, wobei besonders sich die straffen von der Spina nasalis posterior entspringenden Fasern (Azygos uvulae) markiren; die Spitze der Uvula habe ich nicht dabei zu Gesicht bekommen, nur der Hauptstamm des Zäpfchens trat deutlich durch seitliche Furchen hervor, die von seinen Seitenrändern und den angrenzenden Velumtheilen gebildet waren. Eine dorsale Rinne auf der Urula, wie sie Gentzen gesehen hat, konnte ich bei meiner Patientin nicht bemerken.

Betrachtete ich das Bild von oben her bei hintenübergebeugtem Kopfe der Patientin und von oben einfallendem Licht, so war der Eindruck allerdings ein derartiger, dass man kaum einen treffenderen Vergleich als den von Voltolini angeführten finden kann, nehmlich den mit dem Sphincter ani ${ }^{1}$ ). Der Abschluss des Nasen-

1) l. c. S. 208.

Archiv f. pathol. Anat. Bd. LXXIX. Hft. 3. 
rachenraumes ist ein ganz vollständiger, wenn trotzdem geübte Raucher durch Herunterschlucken des Rauches einen Theil desselben aus der Nase hervortreten lassen können, so ist das eben ein besonderes Kunststück, das eine Abweichung des Mechanismus verlangt und nichts gegen die Regel beweist. - Wenn ein Schluck Wasser von der Patientin genommen wurde, so sah ich das Gaumensegel steigen, die hintere Rachenwand vor-, die Seitenwände einander näherrücken, die spincterartige Contraction eintreten, darauf Entfalten derselben und Zurücktreten der betheiligten Glieder der Kette in ihre Ruhestellung; schluckte Patientin aber etwas, was ihr schwerer wurde, in den Oesophagus zu befördern, wie z. B. sehr gut beim Herunterwürgen von zähen Schleimmassen zu. sehen war, so trat eine leichte aber lange nicht vollständige Lockerung der Contraction ein, dann wieder stärkere Zusammenziehung so lange, bis der erwünschte Erfolg erreicht war, es trat gewissermaassen ein vorübergehendes Ausruhen ein. Diesen Vorgang illustrirt recht klar die Curve X, hier bedarf es 5 solcher Angriffe, um zum Endziel zu gelangen, an dieser Curve sieht man auch deutlich das Herabsinken des weichen Gaumens über die Ruhestellung hinaus nach Ablauf der Action (diese Curve verläuft von links nach rechts), wie sie von Gentzen ${ }^{1}$ ) gesehen ist, ein Ausholen des Gaumens vor dem Act, wie es schon von Schuh und Fiaux geseben ist, habe ich bei meiner Kranken nicht mit Sicherheit bemerkt, ich müsste denn den Aufang von Curve VIII, die von rechts nach links gezeichnet ist, so deuten, von diesem Striche weiss ich aber nicht genau, ob er nicht durch eine unpassende Bewegung der Patientin hineingekommen ist.

Eigentlich fehlt zum Abschluss dieser Arbeit noch die Beschreibung der Tubenbewegungen, dies Gebiet war mir aber zu fremd, als dass ich daruber in der surzen Zeit, die mir die Patientin, der meine Studien doch gerade anfingen langweilig zu werden, noch dazu liess, hätte exacte Beobachtungen anstellen können; ausserdetn stimmten die Verbältnisse, wie ich sie bei meiner Patientin sah, durchaus nicht mit den mir bekannten Abbildungen von $\mathrm{Z}$ a u fal und Voltolini äberein. Ich konnte bei Seitwärtsbewegung des Kopfes der Patientin oder auch mit Hülfe eines kleinen Kehlkopt- 
spiegels den Ansatz des Levator veli verfolgen, von hier aus ging eine schlitzartige, ziemlich senkrecht stehende Bucht nach oben, die sich nahe an der lateralen Grenze der hinteren Rachenwand befand, ähnlich wie die Rosenmüller'sche Grube von Zaufal ${ }^{1}$ ) gezeichnet ist, die Hakenfalte konnte ich nicht finden, der Tubenwulst war mit dem Spiegel als eine sehr undeutliche Erhebung zu sehen und war das Ostium pharyngeum tubae selbst nach vorn vollkommen durch den Levator (Levatorwulst) verdeckt und zwar stärker als in Zaufal's Fig. 2, die ,eine Mittellage zwischen Phonation und Schlingbewegung darstellt". Auch mit dem Spiegel gesehen war das Ostium tubae so weit unkenntlich, dass ich erst durch Einführen des Ohrkatheters und Einblasen von Licht mich von seiner Existenz überzengen musste, von Narben war, wie schon oben erwähnt, keine Spur vorbanden, das Gehör der Patientin war auch normal zu nennen. Hatte ich nun mit dem Katheter das Ostium weiter klaffend und besser sichtbar gemacht, so sah ich wie es beim Sprechen sowohl als beim Schlucken vom Velum verschlossen und gleichzeitig nach oben, hinten und innen dislocirt wurde, weitere Angaben bin ich aber nicht zu machen im Stande, da Patientin noch selbigen Tages die Klinik verliess. Ob es sich da um entzündliche Schwellung oder Bildungsanomalie gebandelt hat, muss ich dahin gestellt sein lassen.

1) Archiv f. Ohrenbeilkunde Bd. IX. Taf. VIII u. IX. Fig. 1 a. 2. 